\title{
Identification of Quantitative Trait Loci Controlling Cold Tolerance at the Reproductive Stage in Yunnan Landrace of Rice, Kunmingxiaobaigu
}

\author{
Luyuan Dai*1,2), Xinghua Lin ${ }^{2)}$, Changrong Ye' ${ }^{1)}$, Kazuo Ise ${ }^{3)}$, Koji Saito ${ }^{4)}$, Akira Kato ${ }^{4)}$, Furong Xu'), \\ Tengqiong $\mathrm{Yu}^{1)}$ and Duanpin $\mathrm{Zhang}^{2)}$ \\ 1) Crop Genetic Resources Institute, Yunnan Academy of Agricultural Sciences, Kunming 650205, China \\ 2) Huazhong Agricultural University, Wuhan 430070, China \\ 3) Japan International Research Center for Agricultural Sciences, 1-1 Owashi, Tsukuba, Ibaraki 305-8686, Japan \\ 4) National Agricultural Research Center for Hokkaido Region, 1 Hitsujigaoka, Sapporo, Hokkaido 062-8555, Japan
}

This study focused on a Yunnan landrace of rice, Kunmingxiaobaigu, to identify quantitative trait loci (QTLs) controlling cold tolerance at the reproductive stage using DNA markers. A linkage map with 16 linkage groups covering $1,354.4 \mathrm{cM}$ with a total of 122 markers was constructed based on an $F_{2}$ population consisting of 250 individuals of a cross between Kunmingxiaobaigu and a Japanese variety, Towada. Nine QTLs were detected by interval mapping in the $F_{2}$ population. The nine QTLs were distributed on chromosomes 1 (2 QTLs), 3, 4, 6 (2 QTLs), 7, 10 and 12. The total variance explained by these nine QTLs was $54.2 \%$, while the variance explained by a single QTL ranged from $5.0 \%$ to $37.8 \%$. The validity of four QTLs on chromosomes 3, 6 (2 QTLs) and 7 was confirmed by single-marker analysis in the $F_{3}$ population and they were designated as $q R C T 3, q R C T 6 a, q R C T 6 b$ and $q R C T 7$, respectively. The QTLs qRCT3, qRCT6a and $q R C T 6 b$ were reported here for the first time. The QTL $q R C T 7$ displayed the largest effect, reaching a value of $20.6 \%$.

Key Words: Oryza sativa L., cold tolerance, Kunmingxiaobaigu, quantitative trait loci (QTLs), reproductive stage.

\section{Introduction}

Rice (Oryza sativa L.) is one of the most important staple crops in the world, especially in Asia. Spikelet fertility and grain yield of common rice varieties decrease significantly if rice plants are exposed to a low temperature at their reproductive stage. Total fertile spikelet percentage (TFSP) has been used as an effective criterion to score the cold tolerance of rice at the reproductive stage, which includes the booting stage and the flowering stage (Dai et al. 1999a, Takeuchi et al. 2001, Tanno et al. 1999, 2000). This criterion has been used in many studies on cold tolerance and in

Communicated by Y. Sato

Received December 15, 2003. Accepted May 13, 2004.

*Corresponding author (e-mail: daily@public.km.yn.cn) cold tolerance breeding.

Results of genetic studies on cold tolerance of rice have led to different conclusions depending on the variety tested and on the developmental stage. Cold tolerance of rice at the reproductive stage has been shown to be controlled by four to seven loci in "Somewake" (Futsuhara and Toriyama 1966) but by only two loci in "Hayayuki" (Nishimura 1995). "Norin-PL8", which was derived from the progeny of a backcross between the tropical japonica variety "Silewah" from Indonesia and the breeding line "Hokkai 241", was found to harbor two loci for cold tolerance (Abe et al. 1989). A study on chromosomal location of quantitative trait loci (QTLs) for cold tolerance at the booting stage in "NorinPL8" indicated that at least two regions on chromosomes 3 and 4 are responsible for the cold tolerance (Saito et al. 1995). Takeuchi et al. (2001) identified three QTLs for cold tolerance using doubled-haploid lines derived from a cross between Akihikari and Koshihikari. These three QTLs were mapped to chromosomes 1, 7 and 11. The QTL on chromosome 7 explained $22.1 \%$ of the total phenotypic variance. Andaya and Mackill (2003) identified QTLs for cold tolerance at the booting stage of the temperate japonica variety "M-202" distributed on chromosomes 1, 2, 3, 5, 7, 9 and 12, whose contribution to phenotypic variance ranged from 11 to $17 \%$.

Rice is sown in March and reaches the reproductive stage in July in Kunming, Yunnan Province, China, where the altitude is about $1,900 \mathrm{~m}$ and the average temperatures in July and August are $19.5^{\circ} \mathrm{C}$ and $19.2^{\circ} \mathrm{C}$, respectively. Kunming is an ideal site to evaluate cold tolerance of rice at the reproductive stage (Dai et al. 1999a). Collaborative studies conducted by researchers from Yunnan Academy of Agricultural Sciences, China and from Japan International Research Center for Agricultural Sciences led to considerable progress in improving the cold tolerance of rice varieties through the evaluation of rice genetic resources and breeding materials in Kunming. Forty-two rice varieties with cold tolerance, which have been grown in fields at altitudes up to 2,200 m (Ise et al. 1999) were eventually developed.

Yunnan Province is also considered to be one of the centers of rice genetic diversity (Cheng 1993). Many rice varieties are grown in the regions at altitudes higher than 1,600 $\mathrm{m}$, even in the fields located at altitudes up to $2,695 \mathrm{~m}$. A 
large number of varieties grown in these regions have been found to be tolerant to low temperature, particularly at the reproductive stage (Dai et al. 1999b, Tanno et al. 1999). Kunmingxiaobaigu, which had been cultivated in Kunming, Yunnan Province for more than 300 years and may be an excellent variety (Cheng 1993), was one of the most tolerant landraces to low temperature not only at the booting stage, but also at the flowering stage while Towada was one of the least tolerant varieties in the evaluation of cold tolerance conducted during the collaborative studies between Japan and China (Horisue et al. 1988). In the present study, we analyzed the QTLs for cold tolerance at the reproductive stage, using the population from a cross between Kunmingxiaobaigu and Towada.

\section{Materials and Methods}

\section{Plant materials and cold tolerance evaluation}

An $\mathrm{F}_{2}$ population consisting of 250 individuals was developed from a cross between temperate japonica varieties, Kunmingxiaobaigu and Towada. The $\mathrm{F}_{2}$ population (abbreviated hereafter as $\mathrm{F}_{2}$ ) was planted in a field of an experimental farm belonging to Yunnan Academy of Agricultural Sciences in Kunming in 1997. Heading date of each plant was recorded and transformed into growing days using the equation $\mathrm{G}=(\mathrm{INT}(\mathrm{Hi})-7) * 31+(\mathrm{Hi}-\mathrm{INT}(\mathrm{Hi})) * 100$, where $\mathrm{G}$ denotes growing days while Hi denotes heading date. $\mathrm{F}_{3}$ lines derived from the $F_{2}$ (abbreviated hereafter as $F_{3}$ ) with 20 plants in each line were planted in 1999 in the same field as that in which the $\mathrm{F}_{2}$ had been planted. The mean TFSP of five panicles from a single plant in the $F_{2}$ population was used to score its cold tolerance at the reproductive stage. The mean TFSP of each line in the $F_{3}$ was used to evaluate the cold tolerance of the $F_{3}$. The methods used were described in detail by Dai et al. (1999a). R-value of the correlation coefficient between growing days and TFSP of plant was calculated using Excel (Microsoft).

\section{DNA extraction and molecular marker analysis}

Approximately equal amounts of leaf tissues from at least 20 plants of each $\mathrm{F}_{3}$ line were sampled and bulked for DNA extraction. DNA was digested with 5 restriction endonucleases (BamHI, DraI, EcoRI, EcoRV and HindIII). Restriction fragment length polymorphism (RFLP) analysis, including digestion, Southern blotting and hybridization, was carried out using the method described by Liu et al. (1997). RFLP markers used in the analysis were supplied by Cornell University (Causse et al. 1994), Rice Genome Project of Japan (Harushima et al. 1998), and National Agricultural Research Center for Hokkaido Region (Saito et al. 1991).

Simple sequence repeat (SSR) markers were also used in the analysis. Those with the prefix RM were developed by Wu and Tanksley (1993), Chen et al. (1997) and Temnykh et al. (2000), while those with the prefix OSR were developed by Akagi et al. (1996). A polymerase chain reaction (PCR) was carried out in $10 \mu \mathrm{l}$ of a reaction mixture containing 0.2 $\mu \mathrm{M}$ of each primer, $100.0 \mu \mathrm{M}$ deoxyribonucleotides, $50 \mathrm{mM}$ $\mathrm{KCl}, 10 \mathrm{mM}$ Tris-Cl (pH 8.3), $1.5 \mathrm{mM} \mathrm{MgCl}_{2}, 0.001 \%$ gelatin, $10 \mathrm{ng}$ template DNA, and 0.2 units of AmpliTaq Gold DNA polymerase. The PCR profile was as follows: $94^{\circ} \mathrm{C}$ for 4 min (denaturation), followed by 45 cycles of $94^{\circ} \mathrm{C}$ for 30 sec, $55^{\circ} \mathrm{C}$ for $30 \mathrm{sec}, 72^{\circ} \mathrm{C}$ for $1 \mathrm{~min}$, and a final extension step at $72^{\circ} \mathrm{C}$ for $7 \mathrm{~min}$. The PCR was performed using the GeneAmp PCR System 9700. An alignment of six microliters was electrophoresed on 4\% NuSieve 3:1 agarose (FMC, Rockland, Maine, USA), and polymorphism was detected by ethidium bromide staining.

\section{Map construction}

A linkage map was constructed using MAPMAKER/ EXP 3.0 (Lincoln et al. 1992a). Linkage groups were generated with a LOD score of 3.0 and a recombination fraction of 0.37 using the "group" command. The order of the linkage groups was determined by using "compare", "try" and "ripple" commands. The "order" and "ripple" commands were used to order all the markers on each linkage group. When linkage groups contained too few markers for the "order" command, the "compare" and "ripple" commands were used.

\section{QTL analysis}

Interval mapping was performed by using MAPMAKER/ QTL 1.1 (Lincoln et al. 1992b). A LOD value above 2.4 was considered to indicate the presence of QTLs because the LOD value of 2.4 corresponds to 0.00397 of the genomewide type I error rate (Zeng 1994, Basten et al. 1997, Piepho 2001). The variance explained by a QTL and the total variance explained by all the QTLs were calculated by using MAPMAKER/QTL 1.1. Single-marker analysis by ANOVA with StatView 5.0 (SAS Institute Inc.) was conducted for confirmation of the validity of the QTLs. The difference between mean TFSPs in genotypic classes of the markers was determined by the t-test.

\section{Results}

\section{DNA markers with polymorphism detected}

A total number of 586 RFLP and 219 SSR markers were used to detect polymorphism between the parents. One hundred and twenty-two RFLP markers $(20.8 \%)$ and 49 SSR markers $(22.4 \%)$ were polymorphic. The rates of polymorphic markers varied with the chromosomes, the rates for chromosomes 2 and 5 (10.0\% and $13.8 \%$, respectively) being the lowest, and the rates for chromosomes 8 and 11 (38.0 $\%$ and $37.7 \%$, respectively) being the highest.

\section{Linkage map}

A linkage map consisting of 16 linkage groups and covering $1354.4 \mathrm{cM}$ with a total number of 122 markers was constructed (Fig. 1). Few markers were observed on chromosomes 2, 5 and 9 owing to the lower polymorphism between 
the parents, and there were several gaps even on the other chromosomes. For example, there was no polymorphic marker between RM220 and R494 on chromosome 1, on which 22 available markers had been screened for polymorphism. We also failed to detect any polymorphic marker between XNpb345 and C563 on chromosome 3, between G122
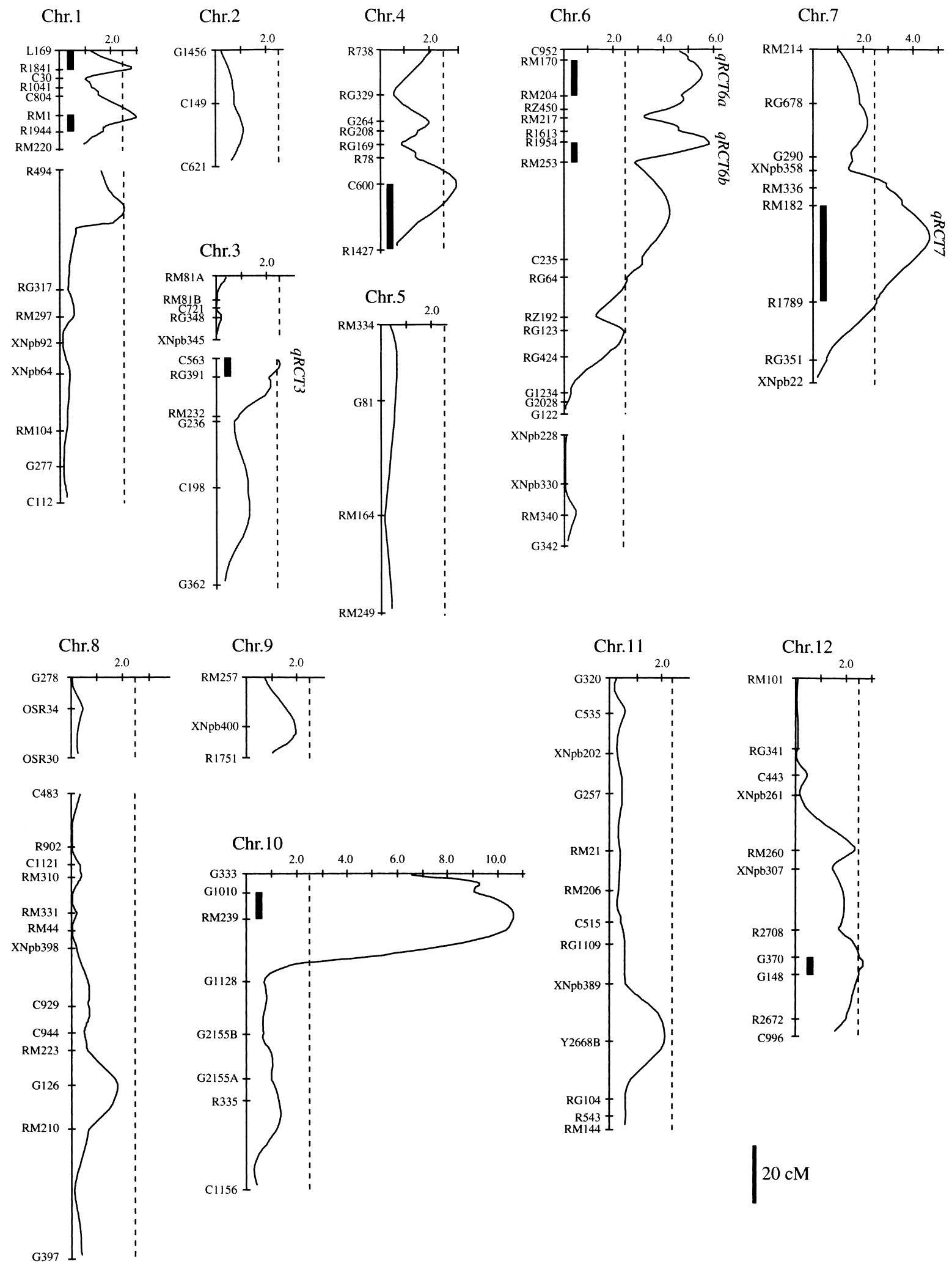

Fig. 1. QTL likelihood map indicating LOD scores for cold tolerance at the reproductive stage. Dotted lines denote the threshold levels of the LOD scores. Bars indicate the intervals at which QTLs for cold tolerance were detected. 
and XNpb228 on chromosome 6 and between OSR30 and C483 on chromosome 8 . The orders of RFLP markers on the map almost coincided with those on published maps (Causse et al. 1994, Harushima et al. 1998).

\section{Phenotypic variation}

The parental varieties, Kunmingxiaobaigu and Towada, are both temperate japonica rice varieties. The $\mathrm{F}_{1}$ plants between the varieties were fertile, suggesting the compatibility of the parents. Cold tolerance at the reproductive stage of Kunmingxiaobaigu is higher than that of Towada (Dai et al. $1999 \mathrm{~b}$ ). The TFSPs of the parents and frequency distribution of the TFSPs in the populations are shown in Table 1 and Figure 2, respectively. The distribution of the TFSPs was

Table 1. TFSPs (means \pm SDs) of the parental varieties and segregating populations

\begin{tabular}{lcc}
\hline \hline \multirow{2}{*}{ Variety } & \multicolumn{2}{c}{ TFSP 1$)$} \\
\cline { 2 - 3 } & 1997 & 1999 \\
\hline Kunmingxiaobaigu & $82.3 \% \pm 6.5$ & $72.7 \% \pm 7.0$ \\
Towada & $5.7 \% \pm 3.7$ & $26.3 \% \pm 7.7$ \\
Populations $\left(\mathrm{F}_{2} / \mathrm{F}_{3}\right)$ & $39.1 \% \pm 22.4\left(\mathrm{~F}_{2}\right)$ & $65.0 \% \pm 11.2\left(\mathrm{~F}_{3}\right)$ \\
\hline \multicolumn{2}{c}{ 1) Total fertile spikelet percentage }
\end{tabular}

A

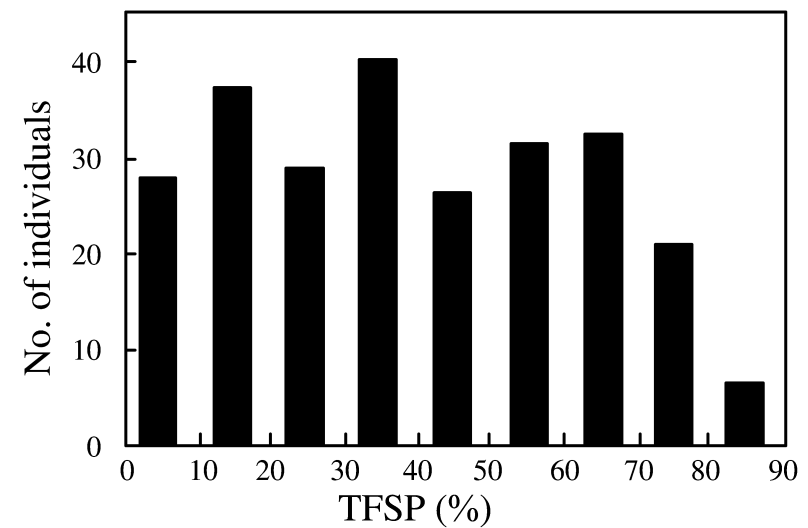

B

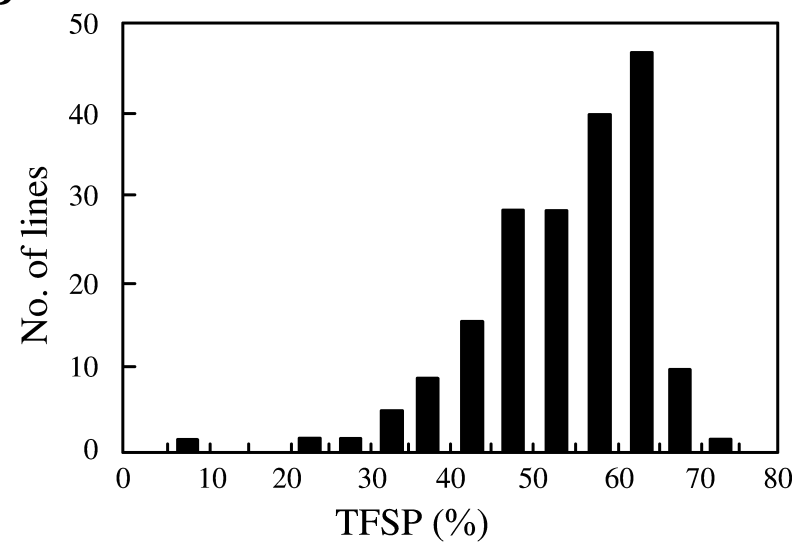

Fig. 2. Frequency distribution of total fertile spikelet percentage (TFSP) in the $\mathrm{F}_{2}$ population (A) and $\mathrm{F}_{3}$ lines (B). continuous, reflecting the quantitative inheritance of the trait. Correlation coefficient between growing days and TFSP in the $F_{2}$ was 0.0195 , showing that TFSP was not correlated with heading date (Fig. 3).

\section{QTL analysis}

Nine QTLs related to cold tolerance in the $\mathrm{F}_{2}$ were detected by interval mapping, as shown in Table 2. The nine QTLs were distributed on chromosomes 1 (2 QTLs), 3, 4, 6 (2 QTLs), 7, 10 and 12 (Fig. 1). The variance explained by a single QTL ranged from 5.0\% to $37.8 \%$. The nine QTLs explained $54.2 \%$ of the total variance in the $\mathrm{F}_{2}$. However, the dominant effect on chromosome 10 indicated that the TFSP of the heterozygotes for the QTL on chromosome 10 was lower than that of the parents. Moreover, the additive effect of the QTL on chromosome 10 was smaller than that of the other QTLs. These results indicate that the QTL on chromosome 10 was not important for cold tolerance.

We conducted single-marker analysis in order to confirm the validity of the QTLs detected on chromosomes 1, 3, 4, 6, 7 and 12 (Table 3). The flanking markers that were significantly associated with TFSP in the $F_{2}$ were used. The markers flanking the QTLs RM170-RM204 on chromosome 6 and RM182-R1789 on chromosome 7 were significantly associated with TFSP also in the $\mathrm{F}_{3}$. As for the QTLs C563RG391 on chromosome 3 and R1954-RM253 on chromosome 6 , one of the flanking markers was significantly associated with TFSP unlike the other was not. However, the markers on chromosomes 1, 4 and 12 were not associated with TFSP in the $\mathrm{F}_{3}$. Based on these results, we concluded that there were 4 QTLs on chromosomes 3, 6 (2 QTLs) and 7, designated $q R C T 3, q R C T 6 a, q R C T 6 b$ and $q R C T 7$, respectively (Fig. 1).

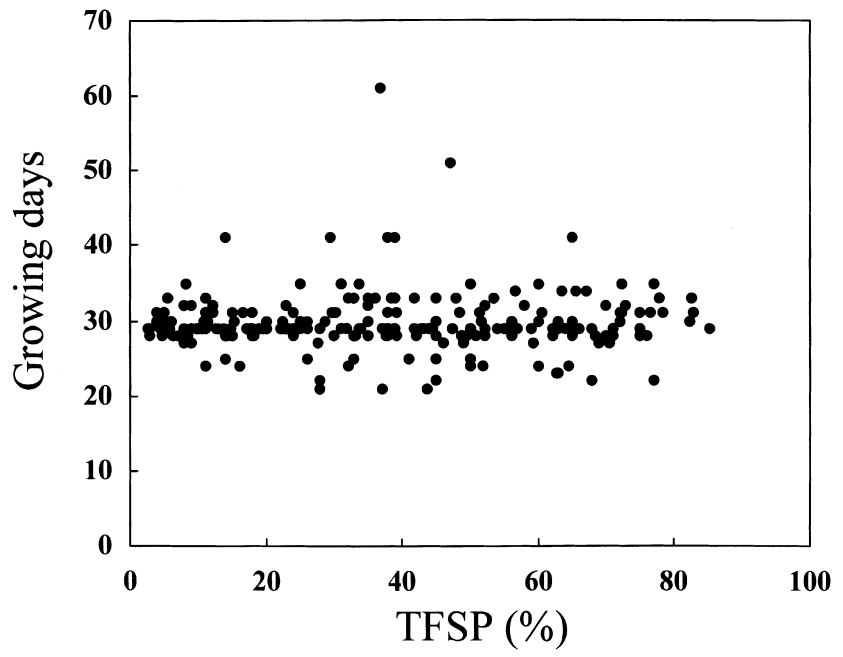

Fig. 3. Correlation between growing days and total fertile spikelet percentage (TFSP) in the $\mathrm{F}_{2}$ population between Kunmingxiaobaigu and Towada. Heading date of each plant was transformed into growing days by the equation $\mathrm{G}=(\mathrm{INT}(\mathrm{Hi})-7) * 31$ $+(\mathrm{Hi}-\mathrm{INT}(\mathrm{Hi})) * 100$, where $\mathrm{G}$ denotes growing days, while Hi denotes heading date. 
Table 2. QTLs conferring cold tolerance detected in the $\mathrm{F}_{2}$ population

\begin{tabular}{clcccc}
\hline \hline Chr. & Flanking Marker & Max LOD & Var \% & Additive & Dominance \\
\hline 1 & RM1-R1944 & 2.96 & 5.4 & 7.54 & 0.07 \\
1 & L169-R1841 & 2.84 & 5.1 & 6.88 & 1.00 \\
3 & C563-RG391 & 2.56 & 5.0 & 7.60 & -3.23 \\
4 & C600-R1427 & 3.07 & 5.5 & 6.73 & 2.59 \\
6 & RM170-RM204 & 5.53 & 11.8 & 10.72 & -1.43 \\
6 & R1954-RM253 & 5.80 & 10.1 & 10.17 & 1.31 \\
7 & RM182-R1789 & 4.64 & 20.6 & 12.18 & -9.93 \\
10 & G1010-RM239 & 10.64 & 37.8 & 5.04 & -28.75 \\
12 & G370-G148 & 2.68 & 5.2 & 7.18 & -0.95 \\
\hline & Total & & 54.2 & & \\
\hline
\end{tabular}

Table 3. Single-marker analysis of the QTLs

\begin{tabular}{lccccr}
\hline \multirow{2}{*}{ Marker } & Chr. & \multicolumn{2}{c}{ TFSP $^{1)}(\%)$} & t-value & $P$ \\
\cline { 3 - 4 } & & $\mathrm{T}$ & $\mathrm{K}$ & & \\
\hline L169 & 1 & 63.9 & 64.2 & 0.136 & 0.8921 \\
R1841 & 1 & 60.9 & 64.2 & 1.295 & 0.1986 \\
RM1 & 1 & 61.2 & 64.7 & 1.176 & 0.2441 \\
R1944 & 1 & 61.6 & 65.3 & 1.519 & 0.1322 \\
C563 & 3 & 63.1 & 68.8 & 2.953 & 0.0041 \\
RG391 & 3 & 64.0 & 67.4 & 1.620 & 0.1094 \\
C600 & 4 & 64.6 & 66.1 & 0.681 & 0.4976 \\
R1427 & 4 & 63.3 & 66.0 & 1.113 & 0.2685 \\
RM170 & 6 & 57.2 & 66.7 & 2.435 & 0.0193 \\
RM204 & 6 & 58.1 & 65.9 & 2.687 & 0.0089 \\
R1954 & 6 & 60.8 & 65.3 & 1.702 & 0.0921 \\
RM253 & 6 & 56.9 & 65.7 & 2.939 & 0.0045 \\
RM182 & 7 & 51.3 & 67.1 & 4.026 & 0.0003 \\
R1789 & 7 & 59.9 & 68.9 & 4.137 & $<0.0001$ \\
G370 & 12 & 63.9 & 66.1 & 0.912 & 0.3643 \\
G148 & 12 & 63.2 & 65.5 & 0.928 & 0.3559 \\
\hline
\end{tabular}

1) Total fertile spikelet percentage. $\mathrm{T}$ and $\mathrm{K}$ denote Towada-type and Kunmingxiaobaigu-type, respectively.

\section{Discussion}

In the sterility caused by low temperature, the growth stages sensitive to cold are the booting stage and the flowering stage (Tanno et al. 2000). However, in previous studies, cold tolerance was analyzed only at the booting stage for technical reasons (Takeuchi et al. 2001, Andaya and Mackill 2003). In the present study, we analyzed the cold tolerance at the reproductive stage, including the booting stage and the flowering stage, of rice growing under the natural cool weather conditions of a highland, and we identified 4 QTLs for cold tolerance on chromosomes 3, 6 and 7 .

Futsuhara and Toriyama (1966) reported that the locus $s d_{2}$ (responsible for ebisu dwarf) was associated with cold tolerance at the booting stage, and $s d_{2}$ has recently been mapped on the short-arm end of chromosome 1. Andaya and Mackill (2003) reported the presence of a QTL for cold tolerance on the short-arm of chromosome 1 . We also detected the QTL on the short arm of chromosome 1, although we could not confirm the validity of the QTL by single-marker analysis in the $\mathrm{F}_{3}$.

We detected the QTL $q R C T 3$ on chromosome 3. Since the closest marker, C563, located on the short arm of chromosome 3 (Harushima et al. 1998), it is possible that qRCT3 located on the short arm. Andaya and Mackill (2003) also detected the QTL qCTB3 on chromosome 3. However, it was mapped in the centromeric region. Therefore, $q R C T 3$ appears to be different from $q C T B 3$.

Andaya and Mackill (2003) reported the existence of the QTL $q C T B 6$, which is close to $q R C T 6 b$. However, $q C T B 6$ is the locus for spikelet development under low temperature conditions. Since spikelet development was not significantly inhibited in our study, it is likely that $q R C T 6 b$ is different from $q C T B 6$. Based on our knowledge, no QTL in the region including $q R C T 6 a$ has been reported. Therefore, the two QTLs on chromosome 6 for cold tolerance were reported here for the first time.

Takeuchi et al. (2001) detected the QTL $q C T-7$ between S1563 and W146 on chromosome 7. We detected the QTL $q R C T 7$ between RM182 and R1789 on chromosome 7. The genetic distance between S1563 and R1789 is $16.9 \mathrm{cM}$ (Harushima et al. 1998), indicating that $q R C T 7$ is close to $q C T-7$. Therefore, it is possible that $q R C T 7$ and $q C T-7$ are the same locus. In our study, it was shown that cold tolerance conferred by $q R C T 7$ is a recessive trait. This is surprising because cold tolerance has generally been considered to be an incompletely dominant trait (Futsuhara and Toriyama 1966). Dominant effect of $q C T-7$ has not been determined because doubled-haploid lines were used in the analysis. The values of phenotypic variance explained by $q R C T 7$ and $q C T-7$ were $20.6 \%$ and $22.1 \%$, respectively, indicating a large effect of the QTLs. Therefore further characterization of QTLs on chromosome 7 would provide useful information for cold tolerance breeding.

The results of QTL analyses of temperate japonica varieties indicated that there is a wide variation in the QTLs for cold tolerance. Moreover, it has been shown that some tropical japonica varieties harbor different QTLs for cold tolerance (Saito et al. 1995). Combining the QTLs based on marker-assisted selection may provide a breakthrough in cold tolerance breeding. 


\section{Acknowledgements}

The authors thank Dr. Zhen Huang and Dr. Jinping Hua for their assistance in the QTL analysis. This research was co-granted by the Yunnan Fund for Sciences and Elite Scientist, Fund of State Key Laboratory for Crop Genetics and Improvement based in Huazhong Agricultural University, and by fellowships of the Japan International Research Center for Agricultural Sciences and Japan Society for the Promotion of Science.

\section{Literature Cited}

Abe,N., S.Kotaka, K.Toriyama and M.Kobayashi (1989) Development of the "RiceNorin PL 8" with high tolerance to cool temperature at the booting stage. Res. Bull. Hokkaido Natl. Agr. Exp. Stn. 152: 9-17 (in Japanese with English summary).

Akagi,H., Y.Yokozeki, A.Inagaki and T.Fujimura (1996) Microsatellite DNA markers for rice chromosomes. Theor. Appl. Genet. 93: 1071-1077.

Andaya,V.C. and D.J.Mackill (2003) QTLs conferring cold tolerance at the booting stage of rice using recombinant inbred lines from a japonica X indica cross. Theor. Appl. Genet. 106: 10841090.

Basten,C.J., B.S.Weir and Z.B.Zeng (1997) QTL cartographer. A reference manual and tutorial for QTL mapping. Version 1.13. (http://statgen.ncsu.edu/).

Causse,M.A., T.M.Fulton, Y.G.Cho, S.N.Ahn, J.Chunwongse, K.Wu, J.Xiao, Z.H.Yu， P.C. Ronald， S.E. Harrington, G. Second, S.R.McCouch and S.D.Tanksley (1994) Saturated molecular map of the rice genome based on an interspecific backcross population. Genetics 138: 1251-1274.

Chen,X., S.Temnykh, Y.Xu, Y.G.Cho and S.R.McCouch (1997) Development of a microsatellite framework map providing genome-wide coverage in rice (Oryza sativa L.). Theor. Appl. Genet. 95: 553-567.

Cheng,K.S. (1993) Rice Genetic Resources in Yunnan. Wu Zhengyi Symposium of seminar on biodiversity inYunnan 1993.12, China, Kunming, Yunnan Presshouse of Science and Technology. p. 90-94 (in Chinese).

Dai,L.Y., C.R.Ye, J.H.Xiong and H.Y.Wang (1999a) Methods for evaluation on cold tolerance of rice. Chin. J. Rice Sci. 13: 62 .

Dai,L.Y., C.R.Ye, F.R.Xu, Y.W.Zeng, B.Liang and G.S.Wen (1999b) Genetic analysis on cold tolerance characteristics of Yunnan rice landrace (Oryza sativa L.) Kunmingxiaobaigu. Chin. J. Rice Sci. 13: 73-76.

Futsuhara,Y. and K.Toriyama (1966) Genetic studies on cool tolerance in rice. III. Linkage relations between genes controlling cool tolerance and marker genes of Nagao and Takahashi. Jpn. J. Breed. 16: 19-30.

Harushima, Y., M.Yano, A.Shomura, M.Sato, T.Shimano, Y.Kuboki, T.Yamamoto, S.Y.Lin, B.A.Antonio, A.Parco, H.Kajiya, N.Huang, K.Yamamoto, Y.Nagamura, N.Kurata, G.S.Khush and T.Sasaki (1998) A high-density rice genetic linkage map with 2275 markers using a single $\mathrm{F}_{2}$ population. Genetics 148 : 479-494.

Horisue, N., Y.Kunihiro, T.Higashi, Z.Oyamada, H.Wang, J.Xiong, S.Zhang, Z.Li and Y.Wang (1988) Screening for cold tolerance of Chinese and Japanese rice varieties and selection of standard varieties. Trop. Agr. Res. Ser. No. 21: 76-87.

Ise,K., Y.Sun and J.Liu (1999) Japan and China joint program for rice breeding using a wide diversity of genetic resources in Yunnan province, China. Breed. Res. 1: 9-13 (in Japanese).

Lincoln, S., M.Daly and E.Lander (1992a) Constructing genetic maps with MAPMAKER/EXP 3.0. Whitehead Institute Technical Report, Cambridge, MA.

Lincoln,S., M.Daly and E.Lander (1992b) Mapping genes controlling quantitative traits with MAPMAKER/QTL 1.1. Whitehead Institute Technical Report, Cambridge, MA.

Liu,K.D., J.Wang, H.B.Li, C.G.Xu, A.M.Liu, X.H.Li and Q.Zhang (1997) A genome-wide analysis of wide compatibility in rice and the precise location of the $S_{5}$ locus in the molecular map. Theor. Appl. Genet. 95: 809-814.

Nishimura,M. (1995) Inheritance of cool tolerance at the booting stage of rice cultivars in Hokkaido. Jpn. J. Breed. 45: 479-485.

Piepho,H.P. (2001) A quick method for computing approximate thresholds for quantitative trait loci detection. Genetics 157: 425-432.

Saito,A., M.Yano, N.Kishimoto, A.Nakagahara, A.Yoshimura, K.Saito, S.Kuhara, Y.Ukai, M.Kawase, T.Nagamine, S. Yoshimura, O.Ideta, R.Ohsawa, Y.Hayano, N.Iwata and M.Sugiura (1991) Linkage map of restriction fragment length polymorphism loci in rice. Jpn. J. Breed. 41: 665-670.

Saito,K., K.Miura, K.Nagano, Y.Hayano-Saito, A.Saito, H.Araki and A.Kato (1995) Chromosomal location of quantitative trait loci for cool tolerance at the booting stage in the rice variety "Norin-PL8". Breed. Sci. 45: 337-340.

Takeuchi, Y., H. Hayasaka, B. Chiba, I. Tanaka, T. Shimano, M. Yamagishi, K.Nagano, T.Sasaki and M.Yano (2001) Mapping quantitative trait loci controlling cool-temperature tolerance at booting stage in temperate Japonica rice. Breed. Sci. 51: 191197.

Tanno,H., J.H.Xiong, L.Y.Dai and C.R.Ye (1999) Some characteristics of cool weather tolerant rice varieties in Yunnan province, China. Jpn. J. Crop Sci. 68: 508-514.

Tanno,H., M.Kinoshita, H.Kiuchi, Y.Hirayama and H.Kikuchi (2000) Estimation of cool-weather tolerance at the flowering stage and its relationship with that of the booting stage in Hokkaido rice varieties. Jpn. J. Crop Sci. 69: 493-499.

Temnykh, S., W.D. Park， N. Aryres， S. Cartinhour， N. Hauck， L. Lipovich, Y.G.Cho, T.Ishii and S.R.McCouch (2000) Mapping and genome organization of microsatellite sequences in rice (Oryza sativa L.). Theor. Appl. Genet. 100: 697-712.

Wu,K.S. and S.D.Tanksley (1993) Abundance, polymorphism and genetic mapping of microsatellites in rice. Mol. Gen. Genet. 241: 225-235.

Zeng,Z.B. (1994) Precision mapping of quantitative trait loci. Genetics 136: $1457-1468$. 